\title{
CORRESPONDENCE
}

\section{Key discoveries often originate with lone researchers}

SIR - Your News Feature 'Group theory' (Nature 455, 720-723;

2008) highlights the dwindling contribution of single authors in the face of the productive increase in collaboration among scientists. Sociologist Brian Uzzi is not alone in questioning whether a lone researcher can still capture the moment when lightning strikes.

Uzzi uses some general metrics to suggest a correlation between the number of citations and the number of authors per paper. But before we rush to embrace these heuristics as a basis for designating best practices for collaboration, our social scientists need a better understanding of how highimpact discoveries in the life sciences come about, for example at different stages of the drugdiscovery process.

Moving along this continuum from early discovery, through the translational stage and on to clinical trials, we would expect to see increases in the aggregate levels of funding and in the number of authors and papers published per year. Consequently, epidemiological evaluation will produce metrics and heuristics that more heavily reflect the practices employed in those later stages of the discovery process.

But social collaboration patterns probably vary with each stage. In the discovery phase, for example, projects are often driven by sole investigators, with support from a tightly knit group of colleagues from universities and biopharmaceutical enterprises, frequently generalists who move readily across disciplines and are at ease with counterintuitive interpretations. These small groups act as the initial nucleating agent, but they are then radically transformed into a complex web of interdependent specialists as the drug, vaccine or biological candidate is developed and moves towards the clinic.

With the explosion of available information, publishers and granting organizations are understandably desperate, seeking tools that more effectively predict high-impact science. But in searching for these tools through the lens of social network analysis, we must not lose sight of the key contribution by a project's pioneer. We shall then see more clearly how collaboration patterns alter as an area of scientific discovery matures.

Readers, reviewers and grant administrators should not be biased against early-stage papers with just one or two authors. Lightning can still strike the solitary explorer whose mind is prepared.

Shawn J. Green, Jon Brendsel PubScholar, 11212 Sorrel Ridge Lane, Oakton, Virginia 22124, USA e-mail: shawn@pubscholar.com

\section{Significant confusion in scientists' grasp of statistics}

SIR - I agree with the points you make about statistical significance under the heading 'Significant' in your News Feature 'Disputed definitions' (Nature 455, 10231028; 2008). However, you do imply that the term 'significant' means simply above or below the $5 \%$ level - a figure chosen by the statistician R. A. Fisher for practical reasons and used in the days when people did arithmetic by hand and referred to printed tables.

Nowadays, of course, personal computers do more general calculations and report probability $(P)$ values directly. A $P$-value may be exact (obtained from counting permutations), an approximation based on asymptotes, or derived from a model by repeated simulation. It then has to be reported and interpreted. Too many scientists - and editors - take the line you reproach and use statistical significance as a criterion of importance.

In addition, significance is calculated in respect of a null model, chosen by the researcher and often in the knowledge that it is untenable. Why would you make measurements to compare groups if you expected to find no differences? A small P-value may therefore be pure fiction as a measure of knowledge gained. This comes on top of any undisclosed history of data selection and of cherry-picking results during the data analysis.

Conversely, numbers obtained from small surveys rarely demonstrate clear-cut (significant) results for individua questions, and a pattern of nonsignificant results in an expected direction across a range of questions could still be worth reporting as indicative. When the null hypothesis is a straw man, it may be more interesting not to be able to demonstrate the anticipated effect — for example, in a pay survey that finds no gender differences.

I endorse your view that what may seem to be sophistry is a crucial distinction. Compare, for example, the statement "The observed differences could occur $5 \%$ of the time if the true effect is zero" with the statement "The probability that the true effect is zero is 5\%". Not only is the latter statement wrong, it does not match the scientific question, which should be to estimate, at a given probability, the minimum size of the effect. Another common variation is to report "no differences between groups" on the basis of $t$-tests that check for a difference only between the group means.

For scientists, talking statistics can be more dangerous than what your interviewee described as "talking Swahili in Louisiana" unless they grasp the grammar as well as the words.

R. Allan Reese Centre for Environment, Fisheries and Aquaculture Science, The Nothe, Weymouth DT4 8UB, UK e-mail: allan.reese@cefas.co.uk

\section{Biocultural diversity should be a priority for conservation}

SIR — In his timely Opinion article on the place of botanic gardens in contemporary society ('Beyond the greenhouse' Nature $\mathbf{4 5 5}$, 596-597; 2008), Mike Maunder notes that gardens are, rightly, "embracing their cultural identity". But it is not enough simply to celebrate the cultural and natural heritage of a place.

As bodies such as Terralingua and the United Nations

Educational, Scientific and Cultural Organization have noted, cultures and languages are becoming as endangered as species and habitats.

Superimposing maps of biodiversity hotspots and areas of cultural and linguistic diversity reveals an extensive degree of overlap, implying that similar threats are common to both (see also W. J. Sutherland Nature 423, 276-279; 2003).

As biological diversity is eroded, key elements of cultural traditions, practices and language are lost. Conversely, as cultures and languages are lost, we lose irreplaceable information about the natural world, as well as notions and philosophies of place, time and humanity.

Biological conservation and cultural conservation must therefore be considered simultaneously within a 'biocultural diversity' framework. In fact, I might argue for the primacy of culture in setting conservation priorities.

Many botanical gardens are developing new education, research and collectionsbased programmes on plant conservation. These can become conservation leaders by explicitly incorporating cultural and linguistic conservation into their programmes and engaging local cultural practitioners.

Christopher P. Dunn Lyon Arboretum, University of Hawai'i, 3860 Mānoa Road, Honolulu, Hawai'i 96822, USA e-mail:cpdunn@hawaii.edu 